\title{
Factores de riesgo para complicaciones cardíacas, obstétricas y neonatales en pacientes con enfermedad cardíaca durante el embarazo
}

\author{
Risk factors for cardiac, obstetric and neonatal complications in patients with heart \\ disease during pregnancy
}

\author{
Edison Muñoz-Ortiz ${ }^{1,2 *}$, Jairo A. Gándara-Ricardo ${ }^{1,2}$, Jesús A. Velásquez-Penagos ${ }^{1,3}$, \\ Natalia Giraldo-Ardila4, Ana M. Betancur-Pizarro', Edwin F. Arévalo-Guerrero', \\ Fernando M. Fortich-Hoyos ${ }^{1}$ y Juan M. Senior-Sánchez ${ }^{1,2}$ \\ ${ }^{1}$ Unidad Funcional Cardiopulmonar y Vascular Periférico, Clínica Cardio-obstétrica, Hospital Universitario San Vicente Fundación; ${ }^{2}$ Departamento \\ de Medicina Interna, Sección de Cardiología; ${ }^{3}$ Departamento de Obstetricia y Ginecología, Sección de Perinatología y Alto Riesgo Obstétrico, \\ Universidad de Antioquia; ${ }^{4}$ Unidad Neonatal, Hospital General de Medellín. Medellín, Colombia
}

\begin{abstract}
Resumen
Objetivo: La enfermedad cardíaca en el embarazo puede ocasionar deterioro clínico y muerte maternofetal. Es indispensable evaluar factores de riesgo relacionados con complicaciones. Método: Estudio observacional y analítico de cohorte retrospectivo con muestra no probabilística por conveniencia de embarazadas con cardiopatía congénita o adquirida, corregida o no, o arritmias que requerían intervención urgente. Se excluyó a pacientes con insuficiencias valvulares leves o moderadas, estenosis valvulares leves, pacientes sin ecocardiografía o sin información del parto. El desenlace fue un compuesto de episodios cardíacos, obstétricos y neonatales. Se realizó análisis univariado y multivariado con modelo de regresión logística y capacidad diferenciadora con área bajo la curva y análisis independiente de la clasificación de riesgo de la OMS modificada (OMSm). Resultados: 104 pacientes con edad promedio de $25 \pm 6.5$ años presentaron episodios cardíacos en 13.5\%, obstétricos en $14.42 \%$ y neonatales en $28.85 \%$. El análisis univariado encontró una relación con el estado funcional de la NYHA, trastornos hipertensivos del embarazo, parto por cesárea, edad gestacional < 27 semanas, hipoxemia y riesgo de la OMSm. En el multivariado sólo el parto por cesárea (OR, 2.68; IC 95\%, 1.05-6.86) y la edad gestacional al momento del parto (OR, 0.39; IC 95\%, 0.22-0.67) mantienen nexo con los desenlaces. El área bajo la curva para el riesgo de la OMSm es de 0.75. Conclusiones: Hay una elevada tasa de efectos adversos en pacientes con enfermedad cardíaca durante el embarazo. La edad gestacional y el parto por cesárea se comportaron como predictores de resultados adversos maternofetales. La clasificación de riesgo de la OMSm tuvo una predicción aceptable de desenlaces adversos.
\end{abstract}

Plabras clave: Embarazo. Cardiopatía. Complicaciones maternas. Complicaciones neonatales.

E-mail: edison.munoz@sanvicentefundacion.com
Disponible en internet: 00-00-2019 Arch Cardiol Mex. 2020;90(2):115-121 www.archivoscardiologia.com 1405-9940/@ 2019 Instituto Nacional de Cardiología Ignacio Chávez. Publicado por Permanyer. Este es un artículo open access bajo la licencia CC BY-NC-ND (http://creativecommons.org/licenses/by-nc-nd/4.0/). 


\section{Abstract}

Objective: Heart disease in pregnancy can cause clinical deterioration and maternal-fetal death. It is essential to evaluate risk factors related to complications. Method: Observational, analytical retrospective cohort study with a non-probabilistic convenience sample of pregnant women with congenital or acquired heart disease, corrected or not, or arrhythmias requiring urgent intervention. Patients with mild or moderate valvular regurgitation, mild valvular stenosis, patients without echocardiography or without delivery information were excluded. The outcome was a composite of cardiac, obstetric and neonatal events. Univariate and multivariate analyzes were performed with logistic regression model and discriminatory capacity with area under the curve and independent analysis of the modified World Health Organization (mWHO) risk classification (mWHO). Results: 104 patients with an average age of $25 \pm 6.5$ years presented cardiac events in $13.5 \%$, obstetric in $14.42 \%$ and neonatal in 28.85\%. The univariate analysis found an association with New York Heart Association functional status, hypertensive disorders of pregnancy, cesarean delivery, gestational age $<27$ weeks, hypoxemia, and mWHO risk. In multivariate only cesarean delivery (odds ratio [OR], 2.68; 95\% confidence interval [Cl], 1.05-6.86) and gestational age at delivery (OR, 0.39; 95\% Cl, 0.22-0.67) maintain association with outcomes. The area under the curve for the mWHO risk is 0.75 . Conclusions: There is a high rate of adverse events in patients with heart disease during pregnancy. Gestational age and cesarean delivery behaved as predictors of adverse maternal-fetal outcomes. The mWHO risk classification had an acceptable prediction of adverse outcomes.

Key words: Pregnancy. Heart disease. Maternal complications. Neonatal complications.

\section{Introducción}

La enfermedad cardiovascular en las pacientes gestantes tiene una prevalencia aproximada de 1 a $4 \%$ y causa 10 a $15 \%$ de la mortalidad materna ${ }^{1,2}$. La gestación representa una presión para el sistema cardiovascular, no sólo por el aumento del $50 \%$ del volumen sanguíneo y el gasto cardíaco, sino por cambios estructurales cardíacos, vasculares y metabólicos ${ }^{3}$. Esto explica el riesgo de descompensación y muerte de las mujeres cardiópatas durante el embarazo. En Colombia, el $39 \%$ de las muertes maternas es efecto de causas indirectas, es decir, aquellas que resultan de una enfermedad anterior al embarazo o que evolucionan durante él y no se relacionan con causas obstétricas; del total de estas causas indirectas, el 3.7\% corresponde a cardiopatías 4 .

En virtud de las características epidemiológicas y socioculturales particulares del país ${ }^{5}$, el patrón de presentación de las enfermedades cardíacas y su efecto sobre la morbimortalidad maternofetal se hace indispensable evaluar los factores relacionados para detectarlos a tiempo.

\section{Metodología}

Estudio observacional y analítico de cohorte retrospectivo con una muestra no probabilística por conveniencia. La muestra consistió en 104 pacientes embarazadas que ingresaron al Servicio de Obstetricia de una institución de alta complejidad, entre el 1 de enero de 2010 y el 31 de diciembre de 2015, incluidas pacientes con cardiopatías congénitas o adquiridas, corregidas o no, y con arritmias que requirieran tratamiento urgente hospitalario. Se revisaron las historias clínicas tanto de la madre como del recién nacido y se empleó un formulario para la recolección de la información en el programa informático Microsoft Excel y con posterioridad se realizó el análisis de los datos con STATA 13. Un cardiólogo, una neonatóloga y un ginecoobstetra corroboraron los desenlaces cardiovasculares, neonatales y obstétricos, respectivamente. Se excluyó a individuos con insuficiencia valvular leve o moderada, a quienes tenían estenosis valvular leve, según los criterios de las guías de enfermedad valvular de la European Society of Cardiology/European Assocation for Cardio-Thoracic Surgery de $2017^{6}$ y a las personas que carecían de estudio ecocardiográfico o de información del parto.

Las variables tomadas de la historia clínica incluyeron datos demográficos como la edad, estado de paridad, edad gestacional al momento de la primera evaluación por el Servicio de Cardiología y al momento del parto, hábitos (tabaquismo y alcohol), comorbilidades de acuerdo con las definiciones de las guías vigentes (hipertensión arterial, diabetes mellitus, dislipidemia y otras afecciones que implicaran adoptar un tratamiento crónico), antecedentes cardiovasculares (intervenciones cardiovasculares anteriores al embarazo quirúrgicas o percutáneas, enfermedad o complicación cardíaca antes del embarazo), saturación de oxígeno al momento del ingreso, presencia de cianosis, fármacos de consumo crónico, con énfasis en anticoagulantes (indicación, 
tipo y seguimiento). De los hallazgos ecocardiográficos se revisó la fracción de expulsión, presencia o no de dilatación del ventrículo derecho y tipo de cardiopatía encontrada. Se verificó la vía del parto y, en caso de parto por cesárea, se revisó si la indicación fue obstétrica o cardíaca.

Los desenlaces hospitalarios maternofetales y obstétricos se catalogaron de la siguiente manera:

- Episodios cardíacos primarios: edema pulmonar, arritmia sintomática sostenida que necesitara tratamiento (taquiarritmia o bradiarritmia), accidente cerebrovascular, paro cardíaco o muerte cardíaca.

- Episodios cardíacos secundarios: deterioro en dos o más clases de la NYHA (New York Heart Association) comparado con la basal, necesidad de procedimiento invasivo cardíaco urgente en el embarazo o posparto intrahospitalario.

- Episodios neonatales: parto prematuro (< 37 semanas de gestación), peso bajo para la edad gestacional (menor al percentil $10^{\circ}$ ), síndrome de dificultad respiratoria, hemorragia intraventricular, muerte fetal ( $\geq 20$ semanas de gestación), o muerte neonatal (hasta los 28 días posparto). Además de los episodios neonatales se investigó la presencia de aborto (<20 semanas de gestación).

- Episodios obstétricos: muerte materna no cardíaca, trastorno hipertensivo relacionado con el embarazo (THRE) y hemorragia posparto $(>500 \mathrm{ml}$ en parto vaginal $0>1,000 \mathrm{ml}$ en cesárea).

\section{Análisis estadístico}

Las variables categóricas se resumen en valores absolutos y relativos, y para las variables continuas se realizó el supuesto de normalidad con la prueba de Shapiro-Wilk. Las variables con distribución normal se resumen con promedios y desviación estándar y las variables que no cumplieron el supuesto de normalidad con medianas y rango intercuartil (RIQ). El desenlace fue un compuesto de episodios cardíacos, obstétricos y neonatales. El análisis univariado se realizó para las variables de riesgo propuestas mediante la $\chi^{2}$, prueba exacta de Fisher o prueba de t para una sola muestra y prueba de Mann-Whitney U para datos continuos. Se consideró un amplio espectro de variables (Tabla 1) para identificar determinantes del desenlace propuesto; los predictores del análisis univariado $(p<0.25)$ se
Tabla 1. Características generales de las pacientes

\begin{tabular}{|c|c|}
\hline Variable & $\mathrm{n}=104$ \\
\hline Edad en años, mediana (rango) & $22(15-40)$ \\
\hline $\begin{array}{l}\text { Número de embarazos previos, } \\
\text { mediana (rango) }\end{array}$ & $2(0-7)$ \\
\hline $\begin{array}{l}\text { Edad gestacional al momento del parto } \\
\text { en semanas, mediana (rango) }\end{array}$ & $34(6-40)$ \\
\hline $\begin{array}{l}\text { Edad gestacional en la primera } \\
\text { evaluación por cardiología en semanas, } \\
\text { mediana (rango) }\end{array}$ & 32 (5 - posparto) \\
\hline $\begin{array}{l}\% \text { de saturación de oxígeno al } \\
\text { ingreso (rango) }\end{array}$ & $94.7(50-100)$ \\
\hline $\begin{array}{l}\text { Antecedente de tabaquismo: número de } \\
\text { pacientes }(\%) \\
\text { Nunca } \\
\text { Extabaquismo } \\
\text { Consumo activo }\end{array}$ & $\begin{array}{l}99(95.1) \\
4(3.85) \\
1(0.96)\end{array}$ \\
\hline $\begin{array}{l}\text { Consumo de alcohol en la gestación: } \\
\text { número de pacientes }(\%) \\
\text { No } \\
\text { Sí }\end{array}$ & $\begin{array}{l}102(98.08) \\
2(1.92)\end{array}$ \\
\hline $\begin{array}{l}\text { Estado funcional clasificado al ingreso: } \\
\text { número de pacientes (\%) } \\
\text { NYHA I } \\
\text { NYHA II } \\
\text { NYHA III } \\
\text { NYHA IV }\end{array}$ & $\begin{array}{c}77-(74.04) \\
17-(16.35) \\
7-(6,73) \\
3-(2.88)\end{array}$ \\
\hline $\begin{array}{l}\text { Riesgo OMS modificado: número de } \\
\text { pacientes }(\%) \\
\text { I } \\
\text { II } \\
\text { III } \\
\text { IV }\end{array}$ & $\begin{array}{l}12(11.54) \\
59(56.73) \\
16(15.38) \\
17(16.35)\end{array}$ \\
\hline $\begin{array}{l}\text { Hallazgos ecocardiográficos } \\
\text { Fracción de expulsión en \% (rango) } \\
\text { Dilatación del ventrículo derecho: } \\
\text { número de pacientes (\%) } \\
\text { No } \\
\text { Sí }\end{array}$ & $\begin{array}{l}80(76.92) \\
24(23.08)\end{array}$ \\
\hline $\begin{array}{l}\text { Comorbilidades: número de } \\
\text { pacientes (\%) } \\
\text { Hipertensión arterial } \\
\text { Hipotiroidismo } \\
\text { Hipertiroidismo } \\
\text { Diabetes mellitus tipo } 2 \\
\text { Enfermedad cerebrovascular } \\
\text { isquémica } \\
\text { Embolia pulmonar } \\
\text { Lupus eritematoso sistémico } \\
\text { Arteritis de Takayasu } \\
\text { Enfermedad mixta del tejido conectivo } \\
\text { Síndrome antifosfolípido } \\
\text { Púrpura trombocitopénica inmunitaria } \\
\text { Epilepsia } \\
\text { Infección por VIH }\end{array}$ & $\begin{array}{c}12(11.5) \\
4(3.8) \\
1(0.96) \\
2(1.92) \\
2(1.92) \\
1(0.96) \\
2(1.92) \\
1(0.96) \\
1(0.96) \\
1(0.96) \\
1(0.96) \\
1(0.96) \\
1(0.96)\end{array}$ \\
\hline
\end{tabular}


Tabla 1. Características generales de las pacientes (Continuación)

\begin{tabular}{|c|c|}
\hline Variable & $n=104$ \\
\hline $\begin{array}{l}\text { Medicamentos de uso crónico: número } \\
\text { de pacientes (\%) } \\
\text { Ninguno } \\
\text { Antagonistas del calcio } \\
\text { Metildopa } \alpha \\
\text { Furosemida } \\
\text { IECA } \\
\text { ASA } \\
\text { Bloqueador } \alpha \\
\text { Levotiroxina } \\
\text { Enoxaparina } \\
\text { Esteroide } \\
\text { Warfarina } \\
\text { ARA-II } \\
\text { Espironolactona } \\
\text { Cloroquina } \\
\text { Azatioprina } \\
\text { Sildenafilo } \\
\text { Hidroclorotiazida } \\
\text { Metformina } \\
\text { Clonidina } \\
\text { Digoxina }\end{array}$ & $\begin{array}{c}75(72.12 \%) \\
5(4.8) \\
5(4.8) \\
5(4.8) \\
5(4.8) \\
5(4.8) \\
4(3.85) \\
4(3.85) \\
4(3.85) \\
3(2.88) \\
3(2.88) \\
1(0.96) \\
1(0.96) \\
1(0.96) \\
1(0.96) \\
1(0.96) \\
1(0.96) \\
1(0.96) \\
1(0.96) \\
1(0.96)\end{array}$ \\
\hline $\begin{array}{l}\text { Indicación de anticoagulación: número } \\
\text { de pacientes }(\%) \\
\text { Ninguna } \\
\text { Válvula cardíaca mecánica } \\
\text { Tromboembolismo venoso } \\
\text { Trombofilia }\end{array}$ & $\begin{array}{c}98(94.23) \\
4(3.85) \\
1(0.96) \\
1(0.96)\end{array}$ \\
\hline $\begin{array}{l}\text { Tipo de anticoagulación: número de } \\
\text { pacientes (\%) } \\
\text { Ninguna } \\
\text { Warfarina } \\
\text { Enoxaparina sin medir valores de } \\
\text { anti-Xa } \\
\text { Warfarina seguida por enoxaparina sin } \\
\text { medir valores de anti-Xa }\end{array}$ & $\begin{array}{l}98(94.23) \\
2(1.92) \\
3(2.88) \\
1(0.96)\end{array}$ \\
\hline
\end{tabular}

ARA-II, antagonistas de los receptores de angiotensina II; ASA: ácido acetilsalicílico; IECA, inhibidores de la enzima convertidora de angiotensina.

incluyeron en un análisis multivariado con un modelo de regresión logística y se determinó su capacidad de discriminación con el área bajo la curva; luego de la regresión logística se configuró un modelo lineal generalizado con función logit para comparar los resultados de los dos métodos alternativos y generar la misma predicción de desenlaces. Se analizó en forma independiente la clasificación de riesgo de la OMS modificada (OMSm) y su capacidad de diferenciación.

\section{Resultados}

Se incluyó a un total de 104 pacientes con promedio de edad de $25 \pm 6.5$ años, con edad gestacional promedio al momento de la evaluación por cardiología de $32 \pm 8$ semanas y el $74 \%$ en estado funcional de la NYHA I (Tabla 1).
Las cardiopatías congénitas fueron el diagnóstico primario más frecuente en las gestantes evaluadas con el $51.92 \%$, seguidas por la enfermedad cardíaca valvular con $25.95 \%$, las arritmias con $15.38 \%$ y la disfunción ventricular izquierda con $4.81 \%$. Otras alteraciones cardíacas menos frecuentes fueron la presencia de hipertensión arterial pulmonar idiopática y los tumores cardíacos con $0.96 \%$ cada uno (Fig. 1). Los diagnósticos cardíacos específicos y su frecuencia de presentación se describen en la tabla 2.

Se presentaron episodios cardíacos primarios en el $13.5 \%$ de los casos y el más frecuente fue el edema pulmonar en el $8.65 \%$ de las pacientes. Los sucesos obstétricos se observaron en el $14.42 \%$ y los neonatales en el $28.85 \%$ de los casos. La vía del parto fue la cesárea en el $50 \%$ de las pacientes; sin embargo, la mayoría tuvo indicaciones obstétricas (39\%) y sólo el $11 \%$ se debió a la cardiopatía.

En el análisis univariado se encontró relación con el desenlace con los siguientes factores: estado funcional de la NYHA, THRE, vía del parto por cesárea, edad gestacional menor de 27 semanas, hipoxemia y clasificación de riesgo de la OMSm (Tabla 3). La edad gestacional tuvo un nexo con desenlace adverso; no obstante, ésta se limitó a los episodios neonatales como era previsible.

En el análisis multivariado sólo dos factores mantienen una vinculación con el desenlace: la vía del parto por cesárea (OR, 2.68; IC 95\%, 1.05-6.86) y la edad gestacional al momento del parto (OR, 0.39; IC 95\%, $0.22-0.67)$. Al ingresar al modelo, las variables estado funcional de la NYHA, multiparidad, dilatación del ventrículo derecho, presión arterial pulmonar, vía del parto, edad gestacional al momento del parto y saturación de oxígeno mostraron una $\chi^{2}$ de $60(p=0.000)$, seudo-R2 de 0.58 y área bajo la curva de 0.93 .

\section{Clasificación de riesgo de la OMS modificada (OMSm)}

La clasificación de riesgo de la OMSm mostró los siguientes resultados: OMSm I con desenlace positivo, 3/12 (25\%); OMSm II, 16/59 (27\%); OMSm III, $10 / 16(62.5 \%)$, y OMSm IV, 16/17 (94\%) ( $\chi^{2}$ de tendencia, 23.21; $p=0.000001)$. Al tomar como referencia la clasificación de riesgo OMSm I (cardiopatías de menor riesgo) y comparar con la clasificación OMSm II, el OR $\mathrm{MH}$ es de 1.11 (IC 95\%, 0.26-4.65); con la OMSm III, el OR MH es de $5(0.95-26.1)$, y con la OMSm IV, el OR MH es de 48 (4.32-532). El área bajo la curva para 


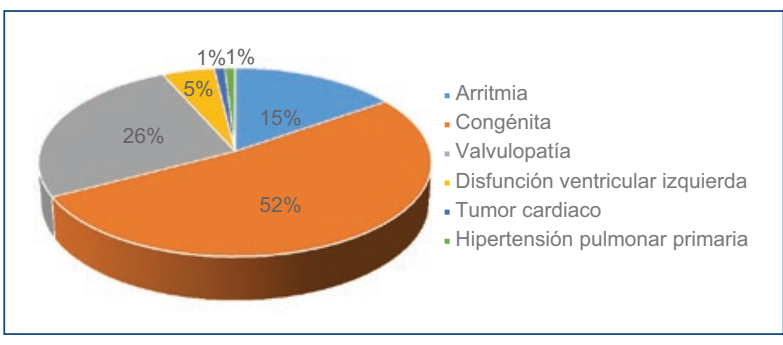

Figura 1. Tipos de cardiopatías encontradas como diagnóstico principal.

diferenciación (curva ROC) es de 0.75 , con adecuada calibración (HL > 0.05) (Fig. 2).

\section{Discusión}

Las pacientes con cardiopatía durante el embarazo tienen un alto riesgo de episodios tanto cardíacos como obstétricos y neonatales, según lo explican no sólo el estrés impuesto por los cambios gestacionales sino el perfil socioeconómico, que incluye en el medio de los autores la consulta tardía a un centro de alta complejidad y la ausencia de consulta preconcepcional. El estado funcional de la NYHA, los THRE, la vía del parto por cesárea, la edad gestacional menor de 27 semanas, la hipoxemia y la clasificación de riesgo de la OMSm se comportan como variables predictoras de sucesos adversos en el análisis univariado, si bien sólo la vía del parto y la edad gestacional conservan su capacidad de predicción en el análisis multivariado. Estas variables tienen una buena capacidad de discriminación (ROC, 0.93) en el modelo de regresión logística; pese a ello, dado el número de desenlaces bajo, debe considerarse sólo exploratorio.

La clasificación de riesgo de la OMSm para cardiopatía y embarazo tiene aceptable capacidad de discriminación (ROC, 0.75), por lo que se confirma que debe ser la escala de riesgo a utilizar en este escenario, además de que permite planear el seguimiento y definir intervenciones tempranas; esto demuestra que cada uno de los grupos tiene un riesgo diferente al compararlo con el primero (OMSm I; $\chi^{2}$ de tendencia), aunque el intervalo de confianza atraviese el 1 en los grupos de riesgo OMSm II y III, explicado por el tamaño de la muestra, que genera intervalos de confianzas poco precisos. Esto es consistente con datos recientes del Registry Of Pregnancy And Cardiac (ROPAC) europeo, en el cual las principales causas del desenlace adverso primario (muerte materna e insuficiencia cardíaca) se presentaron en las pacientes con condiciones OMSm IV, como la hipertensión pulmonar y la miocardiopatía?.
Tabla 2. Diagnósticos cardíacos específicos y frecuencia de presentación

\begin{tabular}{|c|c|}
\hline Variables & $n=104$ \\
\hline Insuficiencia mitral, n (\%) & $11(10.6)$ \\
\hline Conducto arterioso persistente, $\mathrm{n}(\%)$ & $10(9.62)$ \\
\hline Comunicación interventricular, $\mathrm{n}(\%)$ & $10(9.62)$ \\
\hline $\begin{array}{l}\text { Comunicación interventricular con síndrome de } \\
\text { Eisenmenger, } \mathrm{n}(\%)\end{array}$ & $2(1.92)$ \\
\hline $\begin{array}{l}\text { Comunicación interventricular + comunicación } \\
\text { interauricular, } \mathrm{n}(\%)\end{array}$ & $4(3.85)$ \\
\hline $\begin{array}{l}\text { Comunicación interventricular con síndrome de } \\
\text { Eisenmenger + tronco arterioso común, } \mathrm{n}(\%)\end{array}$ & $1(0.96)$ \\
\hline Comunicación interauricular, $\mathrm{n}(\%)$ & $8(7.69)$ \\
\hline $\begin{array}{l}\text { Comunicación interauricular + drenaje venoso } \\
\text { anómalo, } \mathrm{n}(\%)\end{array}$ & $1(0.96)$ \\
\hline $\begin{array}{l}\text { Taquicardia supraventricular por fenómeno de } \\
\text { reentrada, } \mathrm{n}(\%)\end{array}$ & $8(7.69)$ \\
\hline Aorta bivalva sin estenosis o insuficiencia grave, $\mathrm{n}(\%)$ & $6(5.77)$ \\
\hline Disfunción ventricular izquierda, n (\%) & $5(4.81)$ \\
\hline Doble lesión mitral, n (\%) & $5(4.81)$ \\
\hline Válvula mecánica, n (\%) & $3(2.88)$ \\
\hline Anomalía de Ebstein, (\%) & $3(2.88)$ \\
\hline Automatismo ventricular aumentado, $\mathrm{n}(\%)$ & $3(2.88)$ \\
\hline Coartación de aorta corregida, $\mathrm{n}(\%)$ & $3(2.88)$ \\
\hline Bloqueo AV avanzado sin marcapasos previo, $\mathrm{n}(\%)$ & $2(1.92)$ \\
\hline Estenosis mitral, $\mathrm{n}(\%)$ & $2(1.92)$ \\
\hline Estenosis pulmonar congénita, $\mathrm{n}(\%)$ & $2(1.92)$ \\
\hline Estenosis aórtica, n (\%) & $2(1.92)$ \\
\hline Insuficiencia mitral + insuficiencia aórtica, n (\%) & $1(0.96)$ \\
\hline $\begin{array}{l}\text { Transposición congénitamente corregida de grandes } \\
\text { arterias + válvula mecánica + marcapasos, } \mathrm{n}(\%)\end{array}$ & $1(0.96)$ \\
\hline Endocarditis infecciosa, $\mathrm{n}(\%)$ & $1(0.96)$ \\
\hline Válvula protésica biológica, n (\%) & $1(0.96)$ \\
\hline $\begin{array}{l}\text { Presencia de marcapasos por bloqueo AV completo, } \\
\mathrm{n}(\%)\end{array}$ & $1(0.96)$ \\
\hline Insuficiencia aórtica, n (\%) & $1(0.96)$ \\
\hline Tetralogía de Fallot corregida, n (\%) & $1(0.96)$ \\
\hline Hipertensión arterial pulmonar primaria & $1(0.96)$ \\
\hline Taquicardia auricular, $\mathrm{n}(\%)$ & $1(0.96)$ \\
\hline Tetralogía de Fallot no corregida, n (\%) & $1(0.96)$ \\
\hline Fibrilación auricular, n (\%) & $1(0.96)$ \\
\hline Canal AV corregido, $\mathrm{n}(\%)$ & $1(0.96)$ \\
\hline Fibroelastoma, n (\%) & $1(0.96)$ \\
\hline
\end{tabular}


Tabla 3. Factores de riesgo y desenlaces adversos en cardiopatía y embarazo (análisis univariado)

\begin{tabular}{|l|c|c|c|c|c|}
\hline Variable & Desenlace - & Desenlace + & Total & $\mathbf{X}^{2}$ & $\mathbf{p}$ \\
\hline NYHA & 59 & 45 & 104 & 9.98 & 0.019 \\
\hline Embarazo $>2$ & 17 & 19 & 36 & 2.02 & 0.154 \\
\hline Hipertensión arterial durante el embarazo & 0 & 10 & 10 & 14.5 & 0.000 \\
\hline Fracción de expulsión $<40 \%$ & 1 & 2 & 3 & 0.74 & 0.38 \\
\hline Dilatación VD & 11 & 13 & 24 & 1.5 & 0.21 \\
\hline Hipertensión arterial pulmonar & 27 & 28 & 55 & 3.06 & 0.08 \\
\hline Vía del parto Cesárea & 24 & 28 & 52 & 19.6 & 0.000 \\
\hline Edad gestacional $<27$ semanas & 0 & 10 & 10 & 14.5 & $0.0001^{*}$ \\
\hline Hipoxemia & 0 & 5 & 5 & 6.88 & 0.009 \\
\hline Clasificación OMS & 59 & 45 & 104 & 28.21 & 0.000 \\
\hline
\end{tabular}

* Episodios neonatales, $\chi^{2}$ de 93.2, $p=0.000$; episodios obstétricos, $\chi^{2}$ de $0.27, p=0.59$; episodios cardíacos primarios, $\chi^{2}$ de $0.06, p=0.8$.

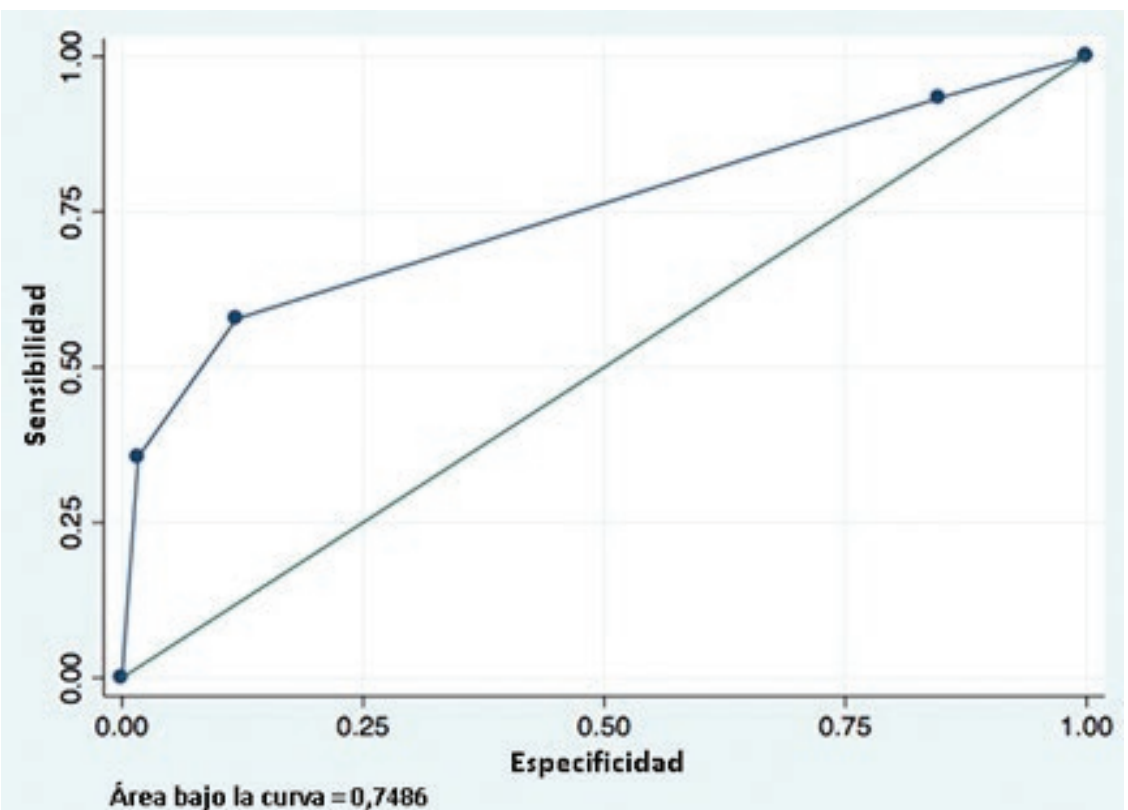

Figura 2. Curva ROC, clasificación de riesgo de la OMS modificada.

En la mayor parte de los casos, la vía de elección para el parto es la vaginal, ya que la cesárea se reserva para indicaciones obstétricas ${ }^{8}$ y para cardiopatías complejas o descompensadas, puesto que atenúa los cambios observados con el trabajo de parto, lo que podría agravar alteraciones como la disección aórtica y la dilatación de la raíz de la aorta mayor de $45 \mathrm{~mm}$, estenosis valvulares graves del lado izquierdo, los casos más graves de hipertensión pulmonar o la insuficiencia cardíaca establecida; en estas condiciones, la cesárea permitiría una mejor vigilancia invasiva y no invasiva ${ }^{1,9}$; sin embargo, se relaciona con mayor riesgo de hemorragia posparto, infección, accidentes quirúrgicos y episodios tromboembólicos, los cuales se incrementan aún más cuando se realiza de manera urgente. Éstas son posiblemente las razones por las cuales persiste como factor predictor en el análisis multivariado en la cohorte descrita. La terminación del 
embarazo, sea vía vaginal o por cesárea, debe planearse y contar con protocolos de atención preestablecidos por un grupo multidisciplinario y con experiencia para mejorar los desenlaces.

La edad gestacional se relaciona con sucesos adversos, en particular neonatales, como cabría esperar por la vulnerabilidad del feto cuando se encuentra lejos del término, según se ha descrito en otras cohortes ${ }^{9,10}$. La elevada tasa de efectos adversos de las pacientes con enfermedad cardíaca durante el embarazo obliga a desarrollar programas multidisciplinarios para la atención de esta población vulnerable, que superen las barreras socioeconómicas, sobre todo en relación con la atención tardía que en promedio ocurrió a las 32 semanas de gestación en esta cohorte, lo cual ha producido un marcador de resultados adversos en un estudio reciente (cuando la primera valoración tiene lugar después de las 20 semanas de edad gestacional) ${ }^{11}$. La actual cohorte desarrollada como preludio de la organización de un grupo cardioobstétrico en un centro de alta complejidad sustenta esta necesidad. Como limitaciones del estudio figuran su naturaleza retrospectiva; sin embargo, el seguimiento de una cohorte prospectiva actual, con intervención más temprana, permitirá cambiar el horizonte y determinar con mayor exactitud los factores predictores de episodios adversos y desarrollar escalas propias ajustadas a la realidad local. Por otro lado, la misma naturaleza retrospectiva propició que no fuera posible realizar escalas que hicieran posible determinar la variabilidad interobservador e intraobservador de los estudios ecocardiográficos, pese a lo cual el servicio de ecocardiografía tiene un seguimiento estricto de las recomendaciones de la American Society of Echocardiography para la obtención de imágenes diagnósticas.

\section{Conclusiones}

Las gestantes con enfermedad cardíaca en esta cohorte presentaron una evaluación tardía, lo que en parte podría llevar a la elevada tasa de efectos adversos. La edad gestacional menor de 27 semanas y el parto por cesárea se comportaron como predictores de resultados adversos maternofetales en el análisis multivariado. Por otro lado, la evaluación independiente de la clasificación de riesgo OMSm tuvo una predicción aceptable de desenlaces adversos y es adecuada para la valoración y planeación del seguimiento de las pacientes. La búsqueda de factores de riesgo que ayuden a predecir los desenlaces adversos en las pacientes embarazadas con enfermedad cardíaca es fundamental para lograr una atención multidisciplinaria de calidad que permita mejorar los resultados.

\section{Conflicto de intereses}

Los autores declaran no tener conflicto de intereses.

\section{Financiamiento}

Unidad de Investigaciones del Hospital Universitario San Vicente Fundación y de la Universidad de Antioquia.

\section{Responsabilidades éticas}

Protección de personas y animales. Los autores declaran que para esta investigación no se han realizado experimentos en seres humanos ni en animales.

Confidencialidad de los datos. Los autores declaran que han seguido los protocolos de su centro de trabajo sobre la publicación de datos de pacientes.

Derecho a la privacidad y consentimiento informado. Los autores declaran que en este artículo no aparecen datos de pacientes.

\section{Bibliografía}

1. Regitz-Zagrosek V, Roos-Hesselink JW, Bauersachs J, Blomstrom-Lundqvist C, Cífková R, De Bonis M, et al. 2018 ESC Guidelines for the management of cardiovascular diseases during pregnancy. The task force for the management of cardiovascular diseases during pregnancy of the European Society of Cardiology (ESC). Eur Heart J. 2018; 39:3165-3241.

2. Sliwa K, Bohm M. Incidence and prevalence of pregnancy-related heart disease. Cardiovasc Res. 2014;101:554-560.

3. Liu X, Arany Z. Maternal cardiac metabolism in pregnancy. Cardiovasc Res. 2014;101(4):545-53.

4. Protocolo de vigilancia epidemiológica de la mortalidad materna. Bogotá: OPS, 2016.

5. Cárdenas LM, Cotes K, Chaparro PE, Niño JA, Paternina A, Castañeda C, et al. Maternal mortality in Colombia 2011: a two level ecological study. PLoS One. 2015;10(3):e0118944.

6. Baumgastner H, Falk V, Bax JJ, De Bomis M, Hamm C, Holm PJ, et al. 2017 ESC/EACTS guidelines for the management of valvular heart disease. The task force for the management of valvular heart disease of the European Society of Cardiology (ESC) and the European Association for Cardio-Thoracic Surgery (EACTS). Eur Heart J. 2017;38:2739-91.

7. Roos-Hesselink J, Baris L, Johnson M, De Backer J, Otto C, Marelli A, et al. Pregnancy outcomes in women with cardiovascular disease: evolving trends over 10 years in the ESC Registry Of Pregnancy And Cardiac disease (ROPAC). Eur Heart J, 2019;[Epub ahead of print].

8. Ruys TP, Cornette J, Roos-Hesslink JW. Pregnancy and delivery in cardiac disease. J Cardiol. 2013;61(2):107-112.

9. Elkayam U, Goland S, Pieper PG, Silverside CK. High risk cardiac disease in pregnancy part I. J Am Coll Cardiol. 2016;68(4):396-410.

10. Drenthen W, Boersma E, Balci A, Moons P, Roos-Hesselink, Mulder BJ, et al. Precitors of pregnancy complicactions in women with congenital heart disease. Eur Heart J. 2010;31(17):2124-2132.

11. Silversides CK, Grewal J, Mason J, Sermer M, Kiess M, Rychel V, et al. Pregnancy outcomes in women with heart disease. The CARPREG II study. J Am Coll Cardiol. 2018;71:2419-30. 